\title{
Plane-wave theory of nondegenerate oscillation in the linear photorefractive passive phase-conjugate mirror
}

\author{
Mark Cronin-Golomb \\ Ortel Corporation, 2015 West Chestnut Street, Alhambra, California 91803
}

Amnon Yariv

California Institute of Technology, Pasadena, California 91125

Received October 25, 1985; accepted January 28, 1986

\begin{abstract}
We present a plane-wave theory of nondegenerate oscillation in the linear passive (self-pumped) phase-conjugate mirror (PPCM). The circumstances under which the plane-wave theory permits spontaneous nondegenerate oscillation in this and other PPCM's are discussed.
\end{abstract}

The observation of frequency-shifted phase-conjugate reflection in photorefractive passive (self-pumped) phase-conjugate mirrors ${ }^{1,2}$ (PPCM's) has given rise to considerable efforts for its theoretical explanation. In this Letter we give a plane-wave theory of oscillation in the linear PPCM, ${ }^{3}$ compare it with the previously developed theory of detuning in the ring PPCM, ${ }^{2}$ and discuss the circumstances in which the plane-wave theory indicates the possibility of nondegenerate oscillation in these devices and in the cat PPCM. ${ }^{4}$

The linear PPCM consists of a photorefractive crystal enclosed by a linear oscillation cavity (Fig. 1). The signal beam, beam 4 , passes through this crystal and pumps oscillation beams 1 and 2 between the cavity mirrors $\mathrm{M}_{1}$ and $\mathrm{M}_{2}$. These self-induced oscillation beams then pump the crystal as a phase-conjugate mirror (PCM) for beam 4 and produce beam 3 , the phase-conjugate reflection. To examine the possibility of nondegenerate oscillation, we analyze the roundtrip phase sum requirements of oscillation in a linear resonator.

The coupled-wave equations describing photorefractive four-wave mixing in the slowly varying field approximation for the transmission grating without linear absorption are

$$
\begin{gathered}
\mathrm{d} A_{1} / \mathrm{d} z=-\gamma g A_{4}, \\
\mathrm{~d} A_{2}{ }^{*} / \mathrm{d} z=-\gamma g A_{3}{ }^{*}, \\
\mathrm{~d} A_{3} / \mathrm{d} z=-\gamma g A_{2}, \\
\mathrm{~d} A_{4}{ }^{*} / \mathrm{d} z=-\gamma g A_{1}{ }^{*},
\end{gathered}
$$

where $\gamma$ is the photorefractive coupling constant, $g=$ $\left(A_{1} A_{4}{ }^{*}+A_{2}{ }^{*} A_{3}\right)$, and $A_{j}$ is the amplitude of beam $j$ normalized by the square root of the conserved total average intensity $I_{0}=I_{1}(z)+I_{2}(z)+I_{3}(z)+I_{4}(z)$. The intensities are defined as $I_{j}=\left|A_{j}\right|^{2}$. To examine the phases more effectively, we separate the amplitudes into magnitude and phase: $A_{j}=I_{j}^{1 / 2} \exp \left(i \varphi_{j}\right)$. We write the round-trip phase-sum rule for the $\mathrm{M}_{1}-\mathrm{M}_{2}$ cavity as

$$
\psi(l)-\psi(0)+2 k L=2 \pi m,
$$

where $m$ is an integer, $L$ is the cavity length, and $\psi(z)=$ $\varphi_{1}(z)-\varphi_{2}(z)$. Since the frequency detunings observed in photorefractive processes are very small $(\approx 1$ $\mathrm{Hz}$ for $1-\mathrm{W} / \mathrm{cm}^{2}$ beams), we have neglected the frequency dependence of the wave number $k$.

The standard theory of the photorefractive effect ${ }^{5,6}$ gives

$$
\gamma=\frac{\omega r_{\mathrm{eff}} n_{0}^{3} E_{q}\left(E_{0}+i E_{d}\right)}{4 c \cos \vartheta\left[E_{0}-\delta \tau\left(E_{d}+E_{\mu}\right)\right]+i\left[E_{d}+E_{q}+\delta \tau E_{0}\right]},
$$

where $\vartheta$ is the angle of incidence of the signal beam and $\delta$ is the oscillation-beam frequency minus the input signal-beam frequency, that is,

$$
\delta=\omega_{1}-\omega_{4}=\omega_{3}-\omega_{2},
$$

where $\omega_{i}$ is the optical frequency of beam $i . E_{o}$ is an externally applied or photovoltaic dc electric field; $E_{\mu}$, $E_{d}$, and $E_{q}$ are internal electric fields characteristic of drift, diffusion, and maximum space charge, respectively. The relevant electro-optic coefficient ${ }^{7}$ is $r_{\text {eff }}$, $n_{0}$ is the crystals refractive index, and $\tau$ is the characteristic time of grating formation and is approximately inversely proportional to the total intensity $I_{0}$. The fact that this time constant depends on the oscillation intensity must be taken into account in the following theory.

To find the relationship between the detuning $\delta$ and

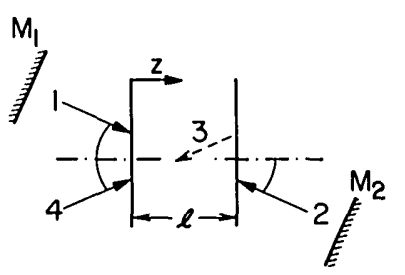

Fig. 1. The linear passive PCM. 


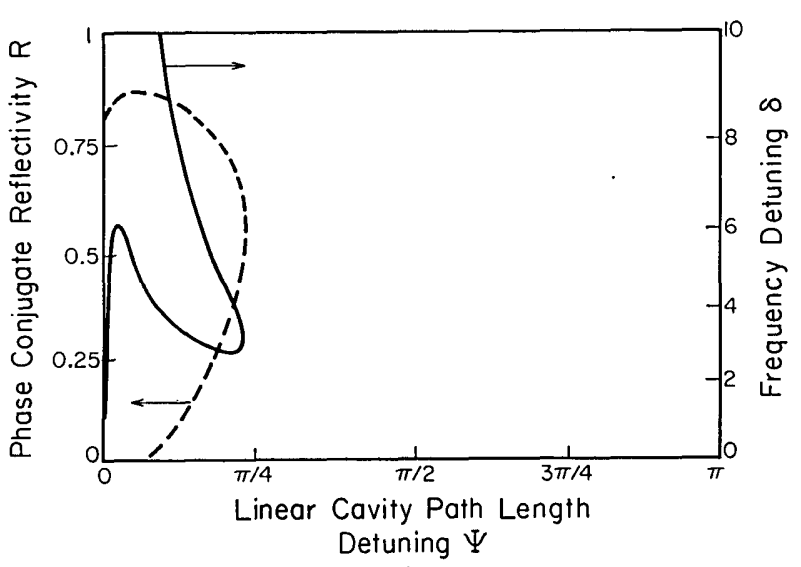

(a)

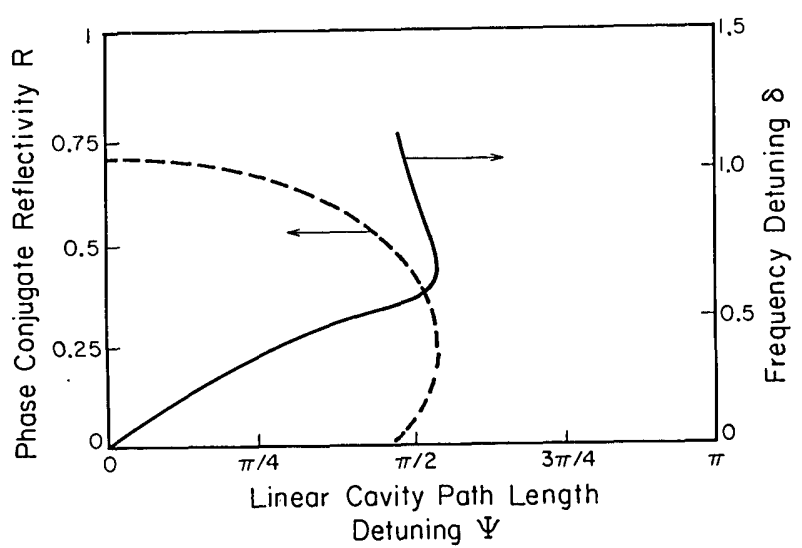

(b)

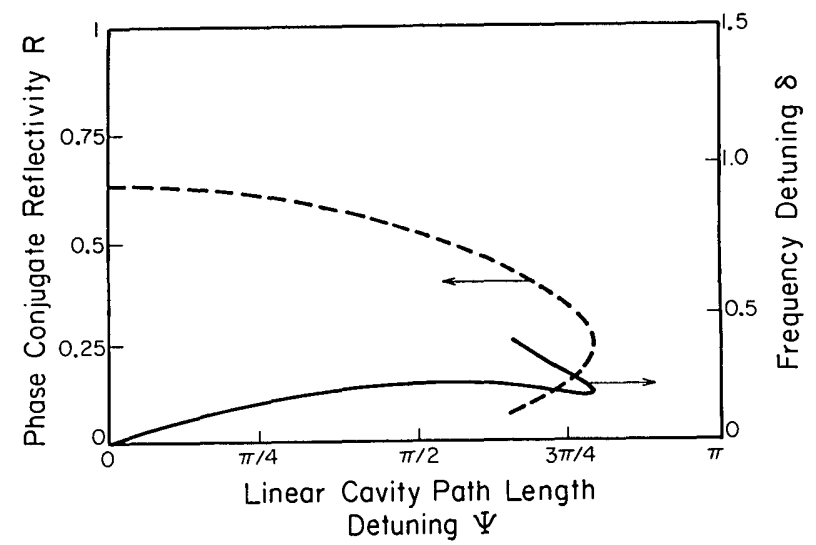

(c)

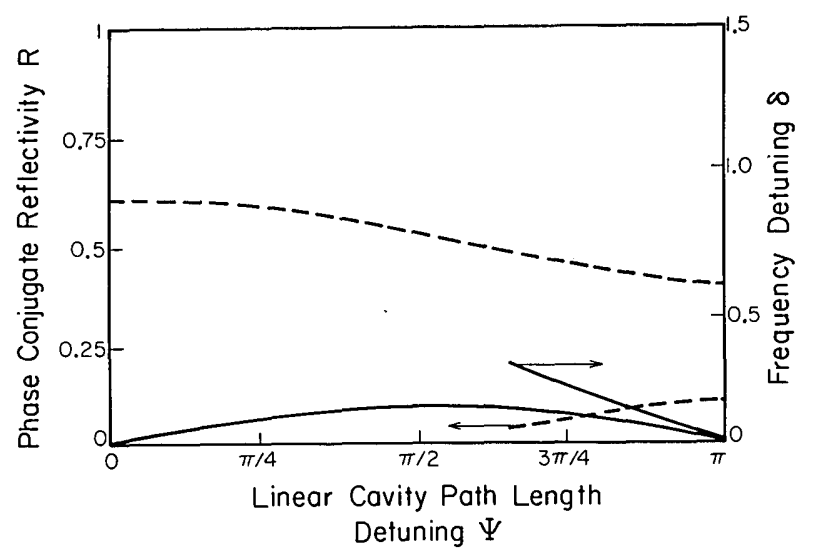

(d)

Fig. 2. Phase-conjugate reflectivity $R$ (dashes) and oscillation-beam frequency detuning $\delta$ versus optical path length $k L$ modulo $2 \pi$ in empty oscillation cavity. Only the path-length range 0 to $\pi$ is shown: the results show natural symmetry about the origin. In each case, the undetuned coupling constant $\gamma l_{0}=-3$ and $M_{2}=0.98$. The detuning $\delta$ is normalized by the constant quantity $I_{4}(0) /\left(I_{0} \tau\right)$. $\mathrm{M}_{1}$ varies as follows: (a) 1.00 , (b) 0.125 , (c) 0.025 , (d) 0.01 . Note the change of scale in $\delta$ for (b), (c), and (d).

the cavity lengthy $L$ as given in Eq. (2), we rewrite Eqs. (1a) and (1b) as

$$
\begin{gathered}
\frac{\mathrm{d} \log A_{1}}{\mathrm{~d} z}=-\gamma I_{4}(1+f), \\
\frac{\mathrm{d} \log A_{2}^{*}}{\mathrm{~d} z}=-\gamma I_{3}(1+1 / f),
\end{gathered}
$$

where

$$
f=A_{2}{ }^{*} A_{3} / A_{1} A_{4}^{*} \text {. }
$$

From the imaginary parts of Eqs. (5), we can derive

$$
\mathrm{d} \psi / \mathrm{d} z=-\operatorname{Im}\left[\gamma I_{4}\left(1+I_{34}+f+I_{34} / f\right)\right],
$$

where $I_{34}=I_{3}(z) / I_{4}(z)$. The quantities $I_{4}, f$, and $I_{34}$ may be readily obtained from the existing theory of the linear PPCM. ${ }^{3}$ First, the intensity flux $\Delta=I_{2}(l)-$ $I_{1}(0)-I_{4}(0)$ is found from the solutions of the equation

$$
M_{1} M_{2}=\left|\frac{T(0)+Q}{\Delta T(0)+Q+(1+\Delta) T(0) / M_{2}}\right|^{2},
$$

where $M_{1}$ and $M_{2}$ are the intensity reflectivities of the cavity mirrors, $T(z)=\tanh [\gamma(l-z) Q / 2]$, and $Q=\left[\Delta^{2}\right.$
$\left.+(\Delta+1)^{2} / M_{2}\right]^{1 / 2}$. Then we have

$$
\begin{gathered}
I_{12}(z) \equiv \frac{I_{1}(z)}{I_{2}(z)}=\frac{1}{M_{2}}\left|\frac{T(z)+Q}{\left[\Delta T(z)+Q+(1+\Delta) T(z)_{\prime} M_{2}\right]}\right|^{2}, \\
I_{34}(z)=\frac{(1+\Delta)^{2}|T(z)|^{2}}{M_{2}|\Delta T(z)+Q|^{2}} \\
I_{4}(z)=\frac{1-\Delta-I_{12}(z)(1+\Delta)}{2\left[1-I_{12}(z) I_{34}(z)\right]} \\
f=-\frac{(\Delta+1) T(z)}{T(z)+Q}\left\{1+\frac{(\Delta+1) T(z)}{M_{2}[\Delta T(z)+Q]}\right\}
\end{gathered}
$$

$\Psi=\psi(l)-\psi(0)$ may then be found by numerical integration of Eq. (7)

Note that if $\gamma$ is real, then $f$ is real, which implies [see Eq. (7)] that $d \psi / d z=0$. In this case, the phases of the oscillation beams are unaffected by the nonlinear interaction and no compensation for cavity length detuning is possible.

Some typical plots of frequency shift $\delta$ and phaseconjugate reflectivity $R$ versus cavity optical path length $k L$ are given in Fig. 2. With $M_{2}=0.98$, we have 
shown what happens as $M_{1}$ is made smaller, approaching the limit of the semilinear PPCM. As this limit is approached, the amount of frequency detuning allowed becomes smaller because the oscillation condition begins not to allow the coupling constant to be complex. Eventually, when $M_{1}=0$, we reach the case of the semilinear PPCM whose solution requires

$$
T(0)+Q=0,
$$

which can be satisfied only if $T(0)$ is real, which implies that $\gamma$ is real, which in the absence of a uniform dc field $E_{0}$ implies that $\delta=0$ [see Eq. (4)] $M_{1}$ is no longer present, and the round-trip phase-sum rule no longer needs to be satisfied. In the semilinear mirror, beam 2 is supplied by retroreflection of beam 1 so that $\omega_{1}=\omega_{2}$. Equation (4) implies then that $\omega_{3}=\omega_{4}$, meaning that no frequency detuning is possible.

We now turn to a discussion of experimental observations of spontaneous nondegenerate oscillation in the linear, ring, and cat PPCM's. Nondegeneracy has been observed in both the ring $\mathrm{PPCM}^{2}$ and the cat PPCM. ${ }^{1}$ Fischer and Sternklar ${ }^{2}$ showed that the plane-wave theory of the ring PPCM indicated that it should exhibit nondegenerate oscillation only if the clockwise path length around the oscillation ring differed from the counterclockwise path length, as would occur if the device were rotating in the case of ringlaser gyroscope or if nonreciprocity in the ring were induced by the Faraday effect in the ring-cavity medium (in their case, an optical fiber). In its conventional interpretation, the cat PPCM is a special case of the ring $P P C M,{ }^{7}$ that is, a ring PPCM containing a double $\mathrm{PCM}^{7}$ in its feedback loop. That the amplitude transmissivity of the double PCM is the same for both beams incident upon it can be shown by the following argument. The quantity $c=A_{1} A_{2}+A_{3} A_{4}$ is independent of $z$, as can be verified by calculating $\mathrm{d} c / \mathrm{d} z$ from Eqs. (1). Since $A_{3}(l)=A_{1}(0)=0$, we have $c=$ $A_{1}(l) A_{2}(l)=A_{3}(0) A_{4}(0)$. Dividing throughout by $A_{4}(0) A_{2}(l)$, we have

$$
\frac{c}{A_{4}(0) A_{2}(l)}=\frac{A_{1}(l)}{A_{4}(0)}=t_{14}=\frac{A_{3}(0)}{A_{2}(l)}=t_{32},
$$

where $t_{14}$ is the left-to-right transmissivity and $t_{32}$ is the right-to-left transmissivity. This device is part of the semilinear PPCM, and, like the semilinear PPCM, requires $\delta=0$. In contrast to the case of the semilinear PPCM, the boundary conditions for a double PCM do not require that $\omega_{1}=\omega_{2}$. The counterpropagating beams in the ring can be detuned from each other, resulting in $\omega_{1}=\omega_{4}, \omega_{3}=\omega_{2}, \omega_{1} \neq \omega_{2}$, and still $\delta=0$. The cat mirror should thus behave in its detuning characteristics exactly like a ring mirror whose feedback is multiplied by the effective transmission due to the double PCM.

Macdonald and Feinberg have pointed out that standard plane-wave theory indicates that the reflectivity of an externally pumped photorefractive PCM can be enhanced by detuning the signal beam from the pumping beams. ${ }^{8}$ They conclude that this enhancement explains spontaneous detuning in the cat
PPCM. We disagree with this conclusion because the same plane-wave theory applied to the conventionally interpreted cat PPCM predicts zero detuning if the ring path lengths are reciprocal and the phase shift between the interference pattern and the refractiveindex grating is $\pi / 2$ for stationary gratings.

Some possible resolutions of the problem of spontaneous detuning in the cat and ring PPCM's are the following:

(1) A non-plane-wave theory may be required to explain the frequency detuning.

(2) The cat PPCM may actually be a linear PPCM, as suggested by existing experimental evidence. ${ }^{9}$ In this case, the plane-wave theory does show that the reflectivity may be enhanced with frequency detuning [see Fig. 2(a)]. However, this possibility does not help to explain spontaneous detuning in the ring PPCM.

(3) The phase shift between the interference pattern and the refractive-index grating may differ from $\pi / 2$ for stationary gratings. Recent experiments indicate that there may in fact be such a departure..$^{10}$

(4) Stimulated scattering processes might be involved. Nondegeneracy, while indeed possible in this case, was not observed in a recent experimental investigation. ${ }^{11}$

In summary, we have presented a plane-wave theory of detuning in the linear PPCM and have related it to previous experimental observations of spontaneous frequency detuning in photorefractive PPCM's.

We would like to acknowledge some helpful discussions with Baruch Fischer. This research was supported by the U.S. Army Research Office, Durham, North Carolina.

\section{References}

1. K. R. MacDonald and J. Feinberg, J. Opt. Soc. Am. A 1, 1213 (1984).

2. B. Fischer and S. Sternklar, Appl. Phys. Lett. 47, 1 (1985).

3. M. Cronin-Golomb, B. Fischer, J. O. White, and A. Yariv, Appl. Phys. Lett. 41, 689 (1982).

4. J. Feinberg, Opt. Lett. 7, 486 (1982). In previous publications, we have called this the two-interaction-region PPCM. The appropriateness of this name is now in doubt, so for the moment we will adopt its colloquial name, in honor of the cat that assisted in its initial demonstration.

5. N. V. Kukhtarev, V. B. Markov, S. G. Odulov, M. S. Soskin, and V. L. Vinetskii, Ferroelectrics 22, 949 (1979).

6. M. Cronin-Golomb, Ph.D. dissertation (California Institute of Technology, Pasadena, Calif., 1983) (unpublished).

7. M. Cronin-Golomb, B. Fischer, J. O. White, and A. Yariv, IEEE J. Quantum Electron. QE-20, 12, (1984).

8. K. R. MacDonald and J. Feinberg, Phys. Rev. Lett. 55, 821 (1985).

9. M. Ewbank and P. Yeh, Opt. Lett. 10, 496 (1985).

10. I. McMichael, P. Yeh, and M. Khoshnevisan, Proc. Soc. Photo-Opt. Instrum. Eng. (to be published).

11. T. Y. Chang and R. W. Hellwarth, Opt. Lett. 10, 408 (1985). 